\title{
Grooming-Enhanced Multicast in Multilayer Networks ${ }^{\dagger}$
}

\author{
Péter Soproni, Marcell Perényi and Tibor Cinkler \\ Department of Telecommunications and Media Informatics, Budapest University of \\ Technology and Economics \\ H-1117, Magyar tudósok körútja 2, Budapest, Hungary \\ \{soproni, perenyim, cinkler\}@tmit.bme.hu
}

\begin{abstract}
In this paper we investigate and evaluate the performance of multicast routing in grooming capable multi-layer optical wavelength division multiplexing (WDM) networks. New wavelength-graph models are proposed for network equipments capable of optical-layer branching of light-paths. We evaluate the cost-effectiveness of electronic and optical-layer multicast and unicast as well. The high scalability of optical multicast against unicast is also showed. All routing and technical constraints are formulated in ILP and realized in our versatile simulator.
\end{abstract}

Keywords: optical multicast, WDM, multilayer network, ILP formulation

\section{Introduction}

In the recent years the traffic load of transport networks has increased significantly due to the rapid growth of Internet and network based applications. There comes a time when the network providers cannot satisfy the traffic demands by merely enlarging network capacities. Many types of demands, responsible for the heavy traffic load, can be regarded as multicast (point to multipoint) demand instead of ordinary unicast (point to point) demand. Applications include digital media broadcasting (e.g. IP-TV, IP-Radio, etc.) or digital media distribution and streaming. Multicast in a transport network is especially useful when the content has high bandwidth requirements, e.g. distribution of one or several digital TV channels from the distributor of the content to the local providers. Other possible application of multicast is described in [1].

There are quite a few papers in the field of optimizing the cost of multicast routing in transport networks. Madhyastha et al. proposed in [2] a heuristic method for the problem of multicast routing and wavelength assignment in WDM ring networks. They proposed new node architectures with electronic or optical Drop-and-Continue (D\&C) feature. The authors of [3] presented an analytical model of grooming problem represented as non-linear programming formulation. They compare the results with heuristic approaches. Several heuristic tree formation algorithms are proposed in [4]. The study assumes a network with sparse splitting capabilities, i.e. only some of the

$\dagger$ This work was supported by the EC within the IST FP6 NoE e-Photon/ONe+ research framework. 
nodes are able to perform splitting of lightpaths in the network. Huang et al. [5] introduce a multicast dynamic light-tree grooming algorithm for solving the problem of formation and maintenance of a multicast tree. The authors of [6] use an ILP formulation to solve the optimal routing and wavelength assignment problem, and show that a network with only a few splitters and wavelength converters can efficiently transfer multicast demands. Two heuristic optimization algorithms are proposed in [7] minimizing the number of allocated wavelengths in the network. The authors aim to perform optimal QoS (Quality of Service) routing and optimal wavelength assignment together.

\section{Problem formulation}

A two-layer network is assumed, where the upper, electronic layer is time switching capable while the lower, optical layer is a wavelength (space) switching capable one. The electronic layer can perform traffic grooming, i.e. multiplexing low bandwidth demands into a single WL channel. The two layers are assumed to be interconnected according to the peer model or vertically integrated according to the multi-region network node framework, i.e., while routing, the control plane has information on both layers and both layers take part in accommodating a demand. Note, that the result is applicable to overlay or augmented interconnection models as well.

The network topology and the number of fibers are assumed given as well as the description of traffic demands. The capacity of WL channels and the cost of routing, (e.g. space switching, optical to electronic conversion, WL branching, etc.) can also be given in advance. We assume static traffic consisting of unicast and multicast traffic demands. A unicast demand has one source, one destination node and a given bandwidth, while a multicast demand has more than one target node. The objective is to reach all destination nodes from the source, while observing all routing and technical constraints.

\section{Network model}

We aimed to use a general network model for routing in two layer networks with grooming and with different types of nodes and arbitrary topologies assuming peermodel that allows optimal routing using the resources of both layers jointly. The model must be able to handle any regular mesh topology. For all these reasons we chose wavelength graph model to represent the network. The WL graph corresponding to the logical network is derived from the physical network considering the topology and capabilities of physical devices.

A simpler version of the model has been first proposed in [8]. ILP formulation of the static RWA problem with grooming and protection has been given in [9].

The types of nodes can also be quite different: Optical Add-and-Drop Multiplexers (OADM), Optical Cross-Connects (OXC: optical core) with full or limited, optical or opto-electrical WL conversion or even an Opto-Electrical Cross-Connect (OEXC: electrical core). Furthermore, some of these nodes support grooming, typically with 
limited number of optical ports. All these properties can be considered in the WL graph model, together with different protection techniques of traffic demands.

The network consists of nodes and links connecting the nodes. Both ends of an optical link (fiber) are attached to an interface (IF) of a physical device. A physical device contains an internal switching fabric and some IFs. The number of available WLs in a fiber is the minimum of the WLs supported by the end IFs. Every link and every physical device has a specific logical representation in the WL graph.

A physical link is derived to as many logical edges as the number of available WLs in the link. The logical sub-graph of a physical device depends on the capabilities of the device. Every edge in the graph has a capacity and a cost of usage. The capacity of the edge usually equals to the WL capacity, which depends on the used carrier (typically $2.5 \mathrm{Gbps}$ - which was assumed in our simulations - or $10 \mathrm{Gbps}$ ). The cost of the edge is determined by its functionality (WL edge, $\mathrm{O} / \mathrm{E}$ conversion, etc.).

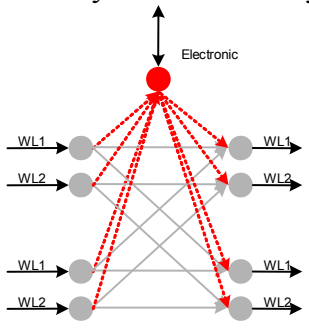

OXC-WL

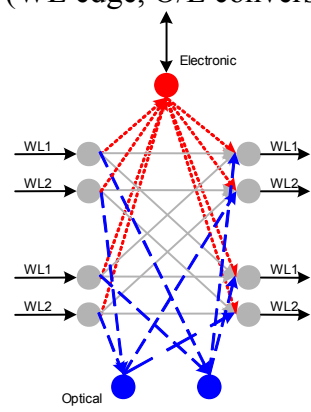

OXC-Wo

Fig. 1. Sub-graph of an OXC-WL device in the wavelength graph (left).

Sub-graph of an OXC-WO device in the wavelength graph (optical splitting capable) (right).

A physical device is modeled by a sub-graph. It represents all IFs of the device, and the capabilities of its internal switching fabric. The WL graph model (together with our ILP framework) can support devices with different capabilities appearing in the network at the same time. The model is easily extendable; type of devices can be changed later, if new internal models are introduced.

A sub-graph of a versatile physical device is depicted in Fig. 1. The equipment is a combination of an OXC with WL-conversion and an OADM: it can originate and terminate traffic demands, as well as perform space-switching. WL-conversion is possible only through the electronic layer. This is illustrated by an electronic node in the sub-graph, while other (pair of) logical nodes correspond to interfaces. Fig. 1 assumes two fibers connected to the device, and two WLs per fiber, which results in two input and two output interfaces - because all edges are directed. We will use this complex node and its extension in the simulations.

\subsection{New node models}

The OXC-WO (OXC with WL-conversion and Optical splitting) devices should be considered as an extension of the OXC-WL type. It introduces a new functionality: 
optical splitting of light-paths. The branching function is represented by dashed edges in the sub-graph. By adding these specific logical edges into the sub-graph, we can accurately determine the cost of optical branching. The in-degree of splitting nodes is always less than or equal to one, while the out-degree is more than or equal to two.

Branching in this device is, however, possible in the electronic layer as well. To accomplish electronic branching, the demand must be first routed up to the electronic layer. The returned (branched) lightpaths should not necessarily use the same WLs. Thus WL-conversion can also be performed in addition to branching (and 3R processing) of the signal.

\section{$5 \quad$ ILP formulations}

We used the following ILP formulation to route multiple multicast trees in the network (Note, optical branching requires a few extra constraints in addition to these): $\mathrm{z}_{\mathrm{ij}}^{\text {or }} \in\{0,1\}$ indicates whether sub-demand $o$ of multicast tree $r$ uses edge $(i, j)$ or not. $\mathrm{x}_{\mathrm{ij}}^{\mathrm{r}} \in\{0,1\}$ indicates whether (end-to-end or multicast demand) $r$ uses edge $(i, j)$. $\mathrm{y}_{\mathrm{ij}} \in\{0,1\}$ indicates if edge $(i, j)$ is used by any of the demands.

$$
\sum_{\forall j \in V_{i}^{+}} z_{j i}^{o r}-\sum_{\forall k \in V_{i}^{-}} z_{i k}^{o r}=\left\{\begin{aligned}
-1 & \text { if } i=s^{r} \\
0 & \text { if } i \notin\left\{s^{r}, t^{\text {or }}\right\} \\
+1 & \text { if } \mathrm{i}=\mathrm{t}^{\text {or }}
\end{aligned}\right.
$$

for every (logical) node $\mathrm{i} \in \mathrm{V}$, tree $r$ and $o$ sub-demand.

$V_{i}^{+}$denotes the set of nodes that can reach node $i$ by the use of one directed edge, while $V_{i}^{-}$is the set of nodes reachable from $i$ by one directed edge. Capitals $A, V, V_{E}$, $O, R$ denote the set of edges ( $\operatorname{arcs})$, nodes (vertices), electronic nodes, sub-demands and the set of multicast trees respectively. The source of tree $r$ is denoted by $s^{r}$, while targets are denoted by $t^{o r}$, where $o$ is the corresponding sub-demand.

$$
\begin{gathered}
\mathrm{z}_{\mathrm{ij}}^{\mathrm{or}} \leq \mathrm{x}_{\mathrm{ij}}^{\mathrm{r}}, \forall(\mathrm{i}, \mathrm{j}) \in \mathrm{A}, \forall \mathrm{o} \in \mathrm{O}, \forall \mathrm{r} \in \mathrm{R} \\
\mathrm{x}_{\mathrm{ij}}^{\mathrm{r}} \leq \sum_{\forall \mathrm{o} \in \mathrm{O}} \mathrm{z}_{\mathrm{ij}}^{\text {or }}, \forall(\mathrm{i}, \mathrm{j}) \in \mathrm{A}, \forall \mathrm{r} \in \mathrm{R} \\
\sum_{\forall \mathrm{j} \in \mathrm{V}_{\mathrm{i}}^{+}} \mathrm{x}_{\mathrm{ji}}^{\mathrm{r}}=\sum_{\forall \mathrm{k} \in \mathrm{V}_{\mathrm{i}}^{-}} \mathrm{x}_{\mathrm{ik}}^{\mathrm{r}} \leq 1, \forall \mathrm{i} \notin \mathrm{V}_{\mathrm{E}}, \forall \mathrm{r} \in \mathrm{R} \\
\sum_{\forall \mathrm{j} \in \mathrm{V}_{\mathrm{i}}^{+}} \mathrm{x}_{\mathrm{ji}}^{\mathrm{r}} \leq\left\{\begin{array}{ll}
0 & \text { if } \mathrm{i}=\mathrm{s}^{\mathrm{r}} \\
1 & \text { if } \mathrm{i} \neq \mathrm{s}^{\mathrm{r}}
\end{array}, \forall \mathrm{i} \in \mathrm{V}_{\mathrm{E}}, \forall \mathrm{r} \in \mathrm{R}\right. \\
\sum_{\forall \mathrm{r} \in \mathrm{R}} \mathrm{x}_{\mathrm{ij}}^{\mathrm{r}} \cdot \mathrm{b}^{\mathrm{r}} \leq \mathrm{B}_{\mathrm{ij}}, \forall(\mathrm{i}, \mathrm{j}) \in \mathrm{A} \\
\mathrm{x}_{\mathrm{ij}}^{\mathrm{r}} \leq \mathrm{y}_{\mathrm{ij}}, \forall(\mathrm{i}, \mathrm{j}) \in \mathrm{A}, \forall \mathrm{r} \in \mathrm{R}
\end{gathered}
$$


Variables:

$$
\begin{gathered}
\mathrm{y}_{\mathrm{ij}} \leq \sum_{\forall \mathrm{r} \in \mathrm{R}} \mathrm{x}_{\mathrm{ij}}^{\mathrm{r}}, \forall(\mathrm{i}, \mathrm{j}) \in \mathrm{A} \\
\sum_{\forall \mathrm{j} \in \mathrm{V}_{\mathrm{i}}^{+}} \mathrm{y}_{\mathrm{ji}}=\sum_{\forall \mathrm{k} \in \mathrm{V}_{\mathrm{i}}^{-}} \mathrm{y}_{\mathrm{ik}} \leq 1, \forall \mathrm{i} \notin \mathrm{V}_{\mathrm{E}}
\end{gathered}
$$

Objective function:

$$
\begin{gathered}
\mathrm{z}_{\mathrm{ij}}^{\mathrm{ro}} \in\{0,1\}, \forall(\mathrm{i}, \mathrm{j}) \in \mathrm{A}, \forall \mathrm{o} \in \mathrm{O}, \forall \mathrm{r} \in \mathrm{R} \\
\mathrm{x}_{\mathrm{ij}}^{\mathrm{r}} \in\{0,1\}, \forall(\mathrm{i}, \mathrm{j}) \in \mathrm{A}, \forall \mathrm{r} \in \mathrm{R} \\
\mathrm{y}_{\mathrm{ij}} \in\{0,1\}, \forall(\mathrm{i}, \mathrm{j}) \in \mathrm{A}
\end{gathered}
$$

(0.1) expresses flow-conservation of each sub-demand of each tree in all nodes. $(0.2)$ tells that edge $(i, j)$ must be allocated if any sub-demand of tree $r$ wants to use it, while $(0.3)$ ensures that edge $(i, j)$ is not allocated in vain. $(0.4)$ ensures that lightpaths cannot branch (or disappear) in non-electronic nodes. Constraint (0.6) says that the aggregate bandwidth of demands using edge $(i, j)$ should be less than the capacity of one WL. (0.7) expresses that a tree can only use an edge, if it is allocated for the routing. In addition (0.8) ensures that edges used by none of the trees are not allocated. (0.8) can be omitted, however, since it is implicitly included in the minimization objective. Constraint (0.9) is similar to (0.4), but on the highest hierarchy-level. It expresses that the number of allocated input and output edges should be equal in non-electronic nodes. The objective function (0.13) expresses that the total cost of allocated edges should be minimized, i.e. we are looking for a minimal-cost routing solution in the network.

The above mentioned formulation can be applied for a mix of unicast and multicast demands as well. The routing problem of solely unicast demands has equivalent ILP formulation using only a two-level hierarchy of variables. Although that formulation contains less variables and inequalities, the solution time does not differ significantly from the solution time of the general formulation. The ILP solver likely recognizes the simplifications and shortcuts, which does not cause significant (more than an order of magnitude) deviation in the solution times.

\section{Technical constraints}

We applied several technical constraints to influence certain properties of the routing. These constraints reflect real-world technical restrictions. Two types of constraints are considered: branching limitations and restrictions concerning size of multicast trees.

\subsection{Branching limitations}

Most of the current switching devices are not able to perform neither optical nor electronic layer branching of the signal due to technical or software restrictions. Thus 
it is necessary to limit the splitting of the signal in both layers. The electronic layer branching can be constrained by the following simple inequality:

$$
\sum_{\forall j \in V_{i}^{-}} x_{i j}^{r} \leq \alpha_{i}, \forall i \in V_{E}, \forall r \in R
$$

The branching limit in node $i$ is denoted by constant $\alpha_{i}$.

The all-optical branching of the signal has further implications. The power of the signal decreases by $3 \mathrm{~dB}$ when it is split in two. In case of splitting in more than two the attenuation is more significant, which impairs correct detection in a receiver.

The number of times the signal is split can be constrained for each sub-demand, because it determines the quality of the signal in the receiver. The number of optical splits can be constrained by the following formula:

$$
\sum_{\forall \mathrm{i} \in \mathrm{V}_{\text {osplit }}} \mathrm{Z}_{\mathrm{ij}}^{\text {or }} \leq \mathrm{L}^{\mathrm{or}}, \forall \mathrm{o}^{\mathrm{r}} \in \mathrm{O}, \forall \mathrm{r} \in \mathrm{R}
$$

where $\mathrm{L}^{\text {or }}$ means the upper limit for sub-demand $o$ of tree $r$.

Constraint (1.2) sets an upper limit for the number of splits along the path of each sub-demand of each tree. The number of optical splits along the path can be calculated by counting the number of edges originated from optical splitting nodes. Note, that we only calculate the total number of splits along the path, not the maximum number of consecutive splits along the path. Although that property is more important, it can be formulated only by too complex linear constraints.

\subsection{Limitation on the size of multicast trees}

Sometimes the optimal solution of a multicast tree produces too long paths between the source and some of the targets. It also implies higher delay, which is unacceptable in some applications or harms the QoS (Quality of Service) agreement. The length (measured in hops in this case) of the path can be limited by the following formula, referred to as depth-limit constraint:

$$
\sum_{\forall(\mathrm{i}, \mathrm{j}) \in \mathrm{A}} \mathrm{z}_{\mathrm{ij}}^{\text {or }} \leq \beta^{\text {or }}, \forall \mathrm{o} \in \mathrm{O}, \forall \mathrm{r} \in \mathrm{R}
$$

Different requirements can be specified for each tree $r$ and sub-demand $o$ by assigning different $\beta^{\text {or }}$ constant values. However, inadequate selection of $\beta^{\text {or }}$ values can render the whole routing problem infeasible. In order to measure the length of the path in some metric such weight factors should be introduced that represent the distance.

It is also possible that size of the whole tree should be limited. The number of links contained by the tree can be restricted by the following formula (tree-size limit):

$$
\sum_{\forall(\mathrm{i}, \mathrm{j}) \in \mathrm{A}} \mathrm{x}_{\mathrm{ij}}^{\mathrm{r}} \leq \mu^{\mathrm{r}}, \forall \mathrm{r} \in \mathrm{R},
$$

$\mu_{\mathrm{r}}$ is a constant value meaning the tree-size limit.

A switching device is called a leaf-node, if it does not branch or relay the multicast tree, but terminates it. It implies that the out-degree of such a node equals to zero. The width of the tree is defined by the number of leaf nodes in it. The breadth-limit of the tree can be set up by the following inequalities: 


$$
\begin{gathered}
\sum_{\forall j \in V_{i}^{-}} x_{i j}^{r} \leq \kappa_{i} \cdot\left(1-v_{i}^{r}\right), \forall i \in t^{r}, \forall r \in R \\
\left(1-v_{i}^{r}\right) \leq \sum_{\forall j \in v_{i}^{-}} x_{i j}^{r}, \forall i \in t^{r}, \forall r \in R \\
\sum_{\forall i \in t^{r}} v_{i}^{r} \leq \eta^{r}, \forall r \in R
\end{gathered}
$$

Variables:

$$
\mathrm{v}_{\mathrm{i}}^{\mathrm{r}} \in\{0,1\}, \forall \mathrm{i} \in \mathrm{V}_{\mathrm{dr}}, \forall \mathrm{r} \in \mathrm{R},
$$

Variable $\mathrm{v}_{\mathrm{i}}^{\mathrm{r}} \in\{0,1\}$ expresses whether tree $r$ is relayed (0) or terminated (1) in node $i$, while $\kappa_{\mathrm{i}}$ is a properly chosen constant of a large value. Constant $\eta^{\mathrm{r}}$ expresses the breadth-limit. The sum in (1.7) should only include the electronic nodes of the target switching devices, since these are the only candidate leaf nodes of the tree. Because of the other routing constraints other leaf nodes (other than the target nodes) are not possible.

\subsection{Soft constraints}

All technical constraints and several of the routing constraints can be reformulated as soft-constraints. It means that a technical or routing recommendation is no longer a member of the ILP constraints; rather it is included in the optimization objective. If a solution does not observe a recommendation, it is not rejected, but penalized. The necessity of a recommendation can be controlled by adjusting the value of the penalty. The advantage of this method is that we can sooner obtain a feasible solution, instead of waiting for a solution observing all the limitations. This technique is especially useful when the whole problem together is infeasible, but lessening the requirements can lead to a feasible solution. For example the soft depth-limit constraint can be expressed by the following formula:

$$
\begin{gathered}
\sum_{\forall(\mathrm{i}, \mathrm{j}) \in \mathrm{A}} \mathrm{z}_{\mathrm{ij}}^{\text {or }} \leq \beta^{\text {or }}+\mathrm{q}^{\text {or }}, \forall \mathrm{o} \in \mathrm{O}, \forall \mathrm{r} \in \mathrm{R} \\
\mathrm{q}_{\mathrm{i}}^{\text {ro }} \in \mathrm{R}^{+}, \forall \mathrm{o} \in \mathrm{O}, \forall \mathrm{r} \in \mathrm{R}
\end{gathered}
$$

$$
\text { Minimize } \sum_{\forall(\mathrm{i}, \mathrm{j}) \in \mathrm{A}} \mathrm{c}_{\mathrm{ij}} \mathrm{y}_{\mathrm{ij}}+\sum_{\substack{\forall \mathrm{o} \in \mathrm{O}, \forall \mathrm{r} \in \mathrm{R}}} \mathrm{c}^{\text {or }} \mathrm{q}^{\text {or }}
$$

$\mathrm{q}^{\text {or }}$ is a positive variable representing the deviation from the recommended value of the limit, while $\mathrm{c}^{\text {or }}$ is a constant meaning the weight of the penalty. Other constraints can be reformulated as soft constraints in the same way.

\section{Results}

In all simulations NRS core network topology [10] was used with 5 WLs per link. 
Fig. 2 shows that multicast routing scales well with the increasing number of both source (trees) and target nodes. The cost curve does not rise beyond a limit, it has a saturation section. It means inserting a new light-tree or further nodes into the current trees has steadily decreasing cost.
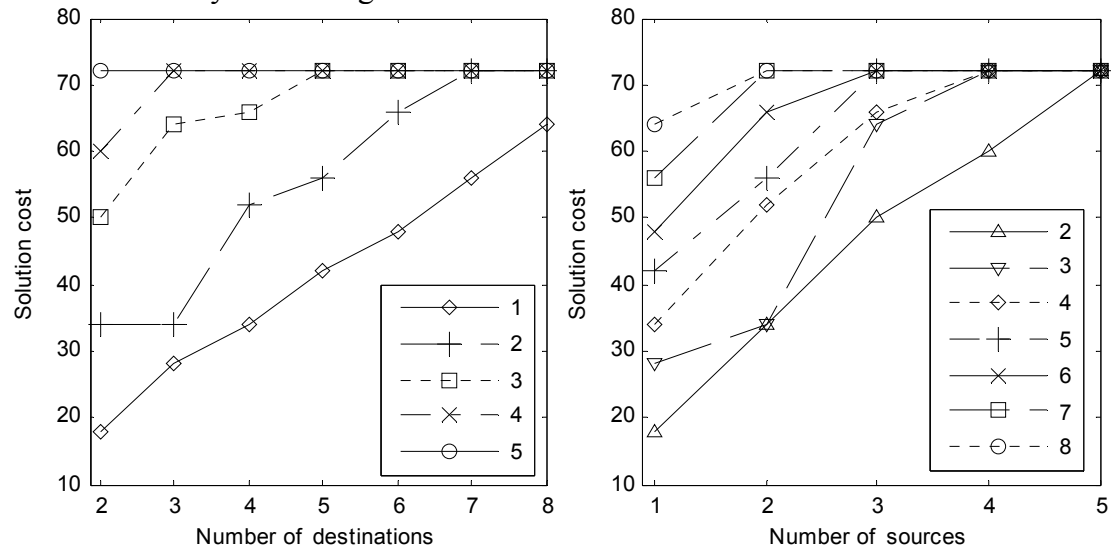

Fig. 2. Cost of routing as a function of the increasing number of targets for different number of sources (left). Cost of routing as a function of the increasing number of sources for different number of targets (right).

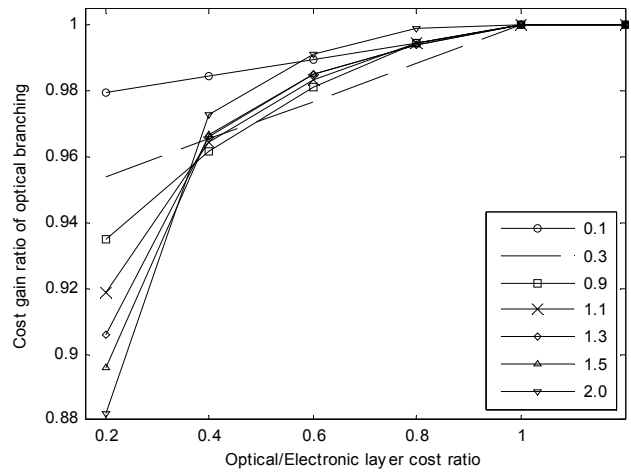

Fig. 3. Cost gain ratio of optical branching versus electronic layer only branching as a function of optical-electronic cost ratio. Different curves assume different WL costs.

Fig. 3 tells that significant cost can be saved if optical layer branching capability is introduced into the network. The figure depicts the cost of routing in an optical branching capable network compared to an only electronic branching capable network. It shows that the lower the price of the optical layer is, the more costs can be saved. This suggests that optical layer branching is particularly worth, if the electronic layer has high cost, which is in accordance with the real-world conditions. The cost gain depends, however, on several properties of the network and the set of demands. 

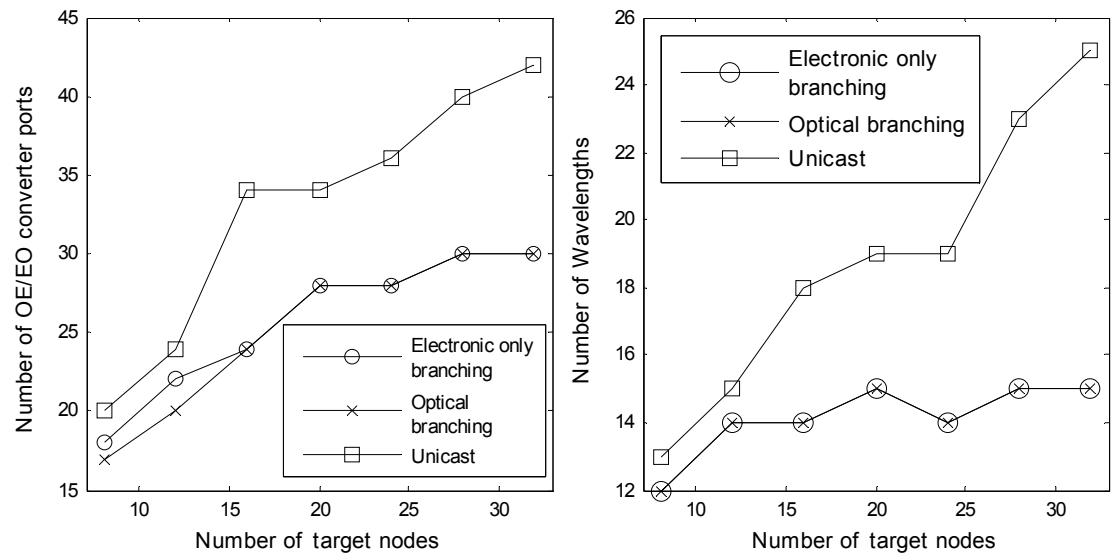

Fig. 4. Required number of converter ports (left) and wavelengths (right) as a function of the number of target nodes for unicast and multicast routing (with and without optical branching).
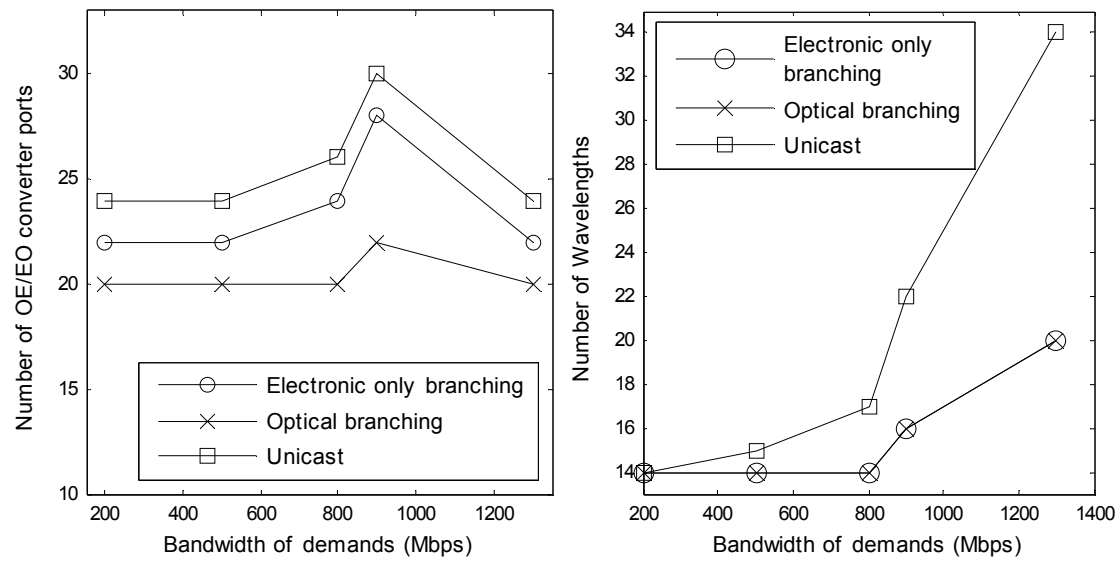

Fig. 5. Required number of converter ports (left) and wavelengths (right) as a function of the bandwidth of demands for unicast and multicast routing (with and without optical branching).

Fig. 4 shows that the required number of converter ports and wavelengths rises with the increasing number of target nodes. Optical branching outperforms electronic branching only if the number of nodes participating in the trees is low - compared to the total number of nodes in the network. As more and more nodes are involved in the trees the advantage of optical branching disappears. It is likely due to the fact that in member nodes of the trees the electronic layer must be reached anyway, thus there is less necessity for optical layer branching in these nodes. Both the required number of converter ports and WLs scale well with the increasing number of demands, unlike in the case of unicast routing, where the required number of resources increases more rapidly. In case of unicast a set of end-to-end demands equivalent to the multicast demands were routed. 
Fig. 5 also shows the required number of converter ports and WLs, but as a function of the increasing bandwidth of the demands. Four trees were routed with three target nodes each. In such a situation optical branching clearly uses less converter ports than electronic branching. However, optical branching does not decrease the required number of WLs. Unicast - naturally - performs far worse in all measurements. Grooming is less and less applicable if the bandwidth of the demands is close to the WL capacity. The sudden fall of the number of OE/EO ports can be explained by this. In case of tiny bandwidths multicast routing does not really show its power, since grooming compensates some of the disadvantages: a huge amount of WLs can be spared by multiplexing tiny demands into a single WL channel.

\section{Conclusion}

In the paper we proposed WL graph models and a new ILP formulation to route unicast and multicast demands in WDM networks. We evaluated the cost and the resource usage of multicast routing with and without optical layer branching of lighttrees and unicast routing as well. We showed that optical branching usually performs better than branching in the electronic layer only. However, if many nodes are involved in the trees then electronic layer is used anyway, and optical branching loses its gain. In case of tiny bandwidths, grooming can well compensate the drawback of unicast over multicast.

\section{References}

1. B. Quinn and K. Almeroth, "IP multicast applications: Challenges and solutions", IETF RFC 3170, Sep. 2001

2. Madhyastha et al., "Grooming of multicast sessions in WDM ring networks", OptiComm 2003: Optical Networking and Communications, Nov. 2003

3. G. V. Chowdhary and C. S. R. Murthy, "Grooming of Multicast Sessions in WDM Mesh Networks", Workshop on Traffic Grooming, 2004

4. X. Zhang et al., "Constrained Multicast Routing in WDM Networks with Sparse Light Splitting", Journal of Lightwave Technology, vol. 18, issue 12, p. 1917, Dec. 2000

5. X. Huang et al., "Multicast Traffic Grooming in Wavelength-Routed WDM Mesh Networks Using Dynamically Changing Light-Trees", Journal of Lightwave Technology, vol. 23 , no. 10 , Oct. 2005

6. D. Yang and W. Liao, "Design of light-tree based logical topologies for multicast streams in wavelength routed optical networks," in Proc. IEEE Information Communications (INFOCOM), San Francisco, CA, Apr. 2003

7. X. H. Jia et al., "Optimization of Wavelength Assignment for QoS Multicast in WDM Networks", IEEE Transactions on Communications, vol. 49, no. 2, Feb. 2001

8. T. Cinkler et al., "Configuration and Re-Configuration of WDM networks", NOC'98, European Conference on Networks and Optical Communications, Manchester, UK, 1998

9. T. Cinkler, "ILP formulation of Grooming over Wavelength Routing with Protection", ONDM 2001, $5^{\text {th }}$ Conference on Optical Network Design and Modeling, Wien, Feb. 2001

10. NRS core network topology (16 nodes, 22 links) http://www.ibcn.intec.ugent.be/css_design/research/projects/INTERNAL/NRS/index.html 